\title{
Extreme Edge Fixed Steiner Graphs
}

\author{
M. Perumalsamy \\ Department of Mathematics \\ Alagappa Chettiar Government \\ College of Engineering and \\ Technology \\ Karaikudi
}

\author{
P. Arul Paul Sudhahar \\ Department of Mathematics \\ Rani Anna Government \\ College for Women \\ Tirunelveli
}

\author{
R. Vasanthi \\ Department of Mathematics \\ Alagappa Chettiar Government \\ College of Engineering and \\ Technology \\ Karaikudi
}

\begin{abstract}
For a non-empty set $W$ of vertices in a connected graph $G$, the Steiner distance $d(W)$ of $W$ is the minimum size of a connected sub graph of $G$ containing $W . S(W)$ denotes the set of vertices that lies in Steiner $W$-trees. Steiner sets and Steiner number of a graph $G$ was studied in [3]. A vertex $v$ is an extreme vertex of a graph $G$ if the sub graph induced by its neighbours is complete. The number of extreme vertices in $G$ is its extreme order $\operatorname{Ext}(G)$. Extreme Steiner graphs were introduced and studied in [7]. Edge fixed Steiner sets of a graph $G$ and the edge fixed Steiner number of $G$ were introduced and characterized in [6]. In this paper we introduce an extreme edge fixed Steiner graph and a perfect extreme edge fixed Steiner graph. Some standard graphs are analyzed and characterized as extreme edge fixed Steiner graphs and perfect extreme edge fixed Steiner graphs. It is shown that for every pair $a, b$ of integers with $2 \leq a \leq b$, there exists a connected graph $G$ with $\operatorname{Ext}(G)=a$ and $s_{e}(G)=b$ for some edge $e$ in $G$.
\end{abstract}

\section{General Terms}

Distance related parameters and Steiner Distance

\section{Keywords}

Steiner set, edge fixed Steiner set, edge fixed Steiner number, extreme edge fixed Steiner set, extreme edge fixed Steiner graph, perfect extreme edge fixed Steiner graph.

\section{INTRODUCTION}

A graph $G=(V, E)$ means a finite undirected connected graph without loops or multiple edges. The order and size of $G$ are denoted by $p$ and $q$ respectively. The distance $d(u, v)$ between two vertices $u$ and $v$ in a connected graph $G$ is the length of a shortest $u v$ path in $G$ [2,4]. For a vertex $v$ of $G$, the eccentricity $e(v)$ is the distance between $v$ and a vertex farthest from $v$. The minimum eccentricity among the vertices of $G$ is called the radius and the maximum eccentricity is called the diameter of $G$ and are denoted by $\operatorname{rad} G$ and $\operatorname{diam} G$ respectively [4]. For basic graph theoretic terminology, Harary [5] is referred.

For a non-empty set $W$ of vertices in a connected graph $G$, the Steiner distance $d(W)$ of $W$ is the minimum size of a connected sub graph of $G$ containing $W$. Necessarily, each such sub graph is a tree and is called a Steiner tree with respect to $W$ or a Steiner $W$-tree. A set $W \underline{V} V$ of vertices in the graph $G$ is called a Steiner set if every vertex in G lies in a Steiner-W-tree which is a minimum connected sub graph of $\mathrm{G}$ containing W. The Steiner number $s(G)$ is the minimum cardinality of a Steiner set [3]. A Steiner set with minimum cardinality is denoted as s-set. A vertex $\mathrm{v}$ is an extreme vertex of a graph $\mathrm{G}$ if the sub graph induced by its neighbours is complete. The number of extreme vertices in $G$ is its extreme order $\operatorname{Ext}(G)$. A graph $G$ is an extreme Steiner graph if $s(G)=\operatorname{Ext}(G)$ [7]. Let $\mathrm{G}$ be a connected graph with at least 3 vertices. For an edge e $=x y$ in $\mathrm{G}$, a set $\mathrm{W} \subseteq \mathrm{V}(\mathrm{G})-\{\mathrm{x}, \mathrm{y}\}$ is called an edge fixed Steiner set of $\mathrm{G}^{\mathrm{if}} \mathrm{W}^{\prime}=\mathrm{W} \cup\{\mathrm{x}, \mathrm{y}\}$ is a Steiner set of $\mathrm{G}$. An edge fixed Steiner set with minimum cardinality is denoted as $\mathrm{s}_{\mathrm{e}}$-set. The minimum cardinality of an edge fixed Steiner set is called the edge fixed Steiner number of $G$ and is denoted by $\mathrm{s}_{\mathrm{e}}(\mathrm{G})$ [6]. In this paper, we introduce an extreme edge fixed Steiner graph and a perfect extreme edge fixed Steiner graph. It is shown that for every pair $a, b$ of integers with $2 \leq \mathrm{a} \leq \mathrm{b}$, there exists a connected graph $\mathrm{G}$ with $\operatorname{Ext}(\mathrm{G})=\mathrm{a}$ and $\mathrm{s}_{\mathrm{e}}(\mathrm{G})=\mathrm{b}$ for some edge e in $\mathrm{G}$.

The following theorems are used wherever required.

Theorem 1.1.[6] For the path $\mathrm{Pp}$ with $\mathrm{p} \geq 3$,

$\mathrm{se}(\mathrm{Pp})=\left\{\begin{array}{c}1 \text { if } \mathrm{e} \text { is an end vertex of } \mathrm{Pp} \\ 2 \text { other wise }\end{array}\right.$

Theorem 1.2.[6] For any complete graph $\mathrm{Kp}$ with $\mathrm{p} \geq 3$, $\mathrm{se}(\mathrm{Kp})=\mathrm{p}-2$ where $\mathrm{e}$ is any edge of $\mathrm{Kp}$

Theorem 1.3.[6] Let $\mathrm{e}=\mathrm{xy}$ be any edge of a connected graph $\mathrm{G}$ of order at least 3 . Then every extreme vertex of $\mathrm{G}$ other than the vertices $\mathrm{x}$ and $\mathrm{y}$ (whether $\mathrm{x}$ and $\mathrm{y}$ are extreme vertices or not) belongs to every edge fixed Steiner set in G. In particular, every end vertex of $\mathrm{G}$ other than $\mathrm{x}$ and $\mathrm{y}$ belongs to every edge fixed Steiner set of G.

Corollory 1.4.[6] Let $\mathrm{T}$ be any non trivial tree and $\mathrm{k}$ be the number of end vertices in $\mathrm{T}$. Let $\mathrm{e}=\mathrm{xy}$ be any edge of $\mathrm{T}$. Then

$s_{e}(T)=\left\{\begin{array}{l}k \text { if neither } x \text { nor } y \text { is an end vertex of } T \\ k-1 \text { if either } x \text { or } y \text { is an end vertex of } T\end{array}\right.$

Theorem 1.5.[6] For any edge e in the wheel

$\mathrm{Wp}=\mathrm{K} 1+\mathrm{Cp}-1(\mathrm{p} \geq 6)$

$s_{e}(W)=\left\{\begin{array}{cr}p-5 & \text { if } e \text { is an edge } \text { e in } C p-1 \\ p-2 & \text { other wise }\end{array}\right.$

Theorem 1.6.[6] If $\mathrm{G}$ is any connected graph of order $\mathrm{p}$ and $\mathrm{e}$ is an edge of $\mathrm{G}$, then $1 \leq \operatorname{se}(\mathrm{G}) \leq \mathrm{p}-2$

Theorem 1.7.[6] No cut - vertex of a connected graph G belongs to any minimum edge fixed Steiner set of G.

Theorem 1.8.[6] For any edge e in the complete bipartite graph $K m, n$ with $m \leq n$ and $m+n \geq 3, \operatorname{se}(K m, n)=m+n-2$.

Theorem 1.9.[4] Every nontrivial connected graph contains at least two vertices that are not cut- vertices. 


\section{EXTREME EDGE FIXED STEINER}

\section{GRAPH}

Definition 2.1. Let $G$ be a simple connected graph with at least three vertices. Let $S_{e x t}$ be the set of all extreme vertices of $G$. For an edge $e=x y$ in $G$, an edge fixed Steiner set W of $G$ is called as an extreme edge fixed extreme edge fixed Steiner set of $G$ if

$$
W=\left\{\begin{array}{c}
s_{\text {ext }} \text { ifneither } x \text { nor } y \text { is an extreme } \\
\text { vertex of } G \\
s_{\text {ext }}-\{x\} \text { or } s_{\text {ext }}-\{y\} \text { according as neither } \\
x \text { nor } y \text { is an extreme } \\
\text { vertex of } G \\
s_{\text {ext }}-\{x, y\} \text { if both } x \text { and } y \text { are extreme } \\
\text { vertices of } G
\end{array}\right.
$$

Remark 2.2. The number of extreme vertices in a graph $G$ is denoted by $\operatorname{Ext}(G)$.

Definition 2.3. Let $G$ be a simple connected graph with at least three vertices. Then $G$ is called an extreme edge fixed Steiner graph if there exists an edge $x y$ in $G$ such that the edge $x y$ has an extreme edge fixed Steiner set of $G$.

Example 2.4. Consider the graph $G$ shown in Figure 1 .

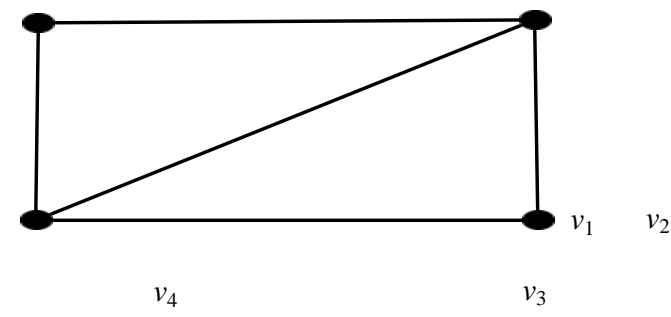

Figure 1: An extreme edge fixed Steiner graph with one edge having extreme edge fixed Steiner set

$s_{e}$-sets for each edge $e$ of $G$, the corresponding edge fixed Steiner number and the set $S_{\text {ext }}$ of extreme vertices of $G$ are given in the Table 1.

Table 1:

\begin{tabular}{|c|c|c|c|c|}
\hline Edge e & se-sets & $\operatorname{se}(\mathrm{G})$ & Sext & $\begin{array}{c}\text { Extreme edge } \\
\text { fixed Steiner set } \\
\text { of G or not }\end{array}$ \\
v1v4 & $\{\mathrm{v} 2, \mathrm{v} 3\}$ & 2 & $\{\mathrm{v} 1, \mathrm{v} 3\}$ & No \\
$\mathrm{v} 2 \mathrm{v} 3$ & $\{\mathrm{v} 1, \mathrm{v} 4\}$ & 2 & $\{\mathrm{v} 1, \mathrm{v} 3\}$ & No \\
$\mathrm{v} 2 \mathrm{v} 4$ & $\{\mathrm{v} 1, \mathrm{v} 3\}$ & 2 & $\{\mathrm{v} 1, \mathrm{v} 3\}$ & Yes \\
$\mathrm{v} 3 \mathrm{v} 4$ & $\{\mathrm{v} 1, \mathrm{v} 2\}$ & 2 & $\{\mathrm{v} 1, \mathrm{v} 3\}$ & No \\
& & & \\
\hline
\end{tabular}

From the Figure 1 and from the Table 1, it is observed that for the edge $e=v_{2} v_{4}$, the vertices of the edge fixed Steiner set are the same as the extreme vertices of the graph $G$ of Figure 1. Therefore the graph in Figure 1 is an extreme edge fixed Steiner graph.

Example 2.5. Consider the graph $G$ shown in Figure 2 .

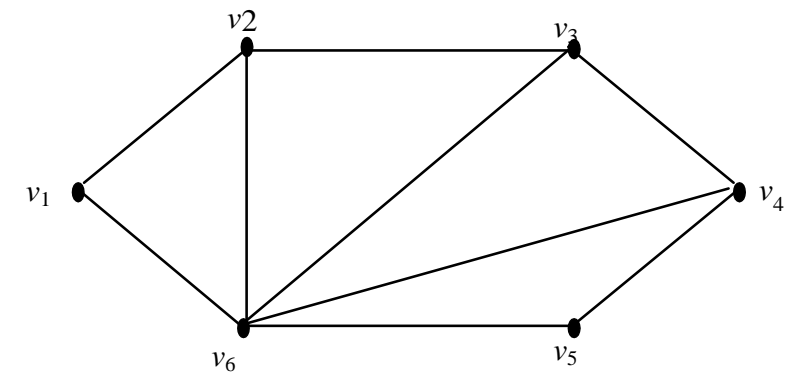

Figure 2: An extreme edge fixed Steiner graph having extreme edge fixed Steiner sets for two edges

$s_{e}$-sets for each edge $e$ of $G$, the corresponding $s_{e}(G)$ and the set $S_{e x t}$ of extreme vertices of $G$ are given in Table 2 .

From Table 2 and from the Figure 2, we observe that two edges have the extreme edge fixed Steiner sets. Therefore the graph shown in Figure 2 is an extreme edge fixed Steiner graph.

Table 2:

\begin{tabular}{|c|c|c|c|c|}
\hline Edge e & $s_{e}$-sets & $s_{e}(G)$ & $S_{\text {ext }}$ & \begin{tabular}{|c|} 
Extreme \\
edge fixed \\
Steiner set \\
of $G$ or not
\end{tabular} \\
\hline$v_{1} v_{2}$ & $\left\{v_{3}, v_{4}, v_{5}\right\}$ & 3 & $\left\{v_{1}, v_{4}, v_{5}\right\}$ & No \\
\hline$v_{2} v_{3}$ & $\left\{v_{1}, v_{4}, v_{5}, v_{6}\right\}$ & 4 & $\left\{v_{1}, v_{4}, v_{5}\right\}$ & No \\
\hline$v_{3} v_{4}$ & $\left\{v_{1}, v_{5}\right\}$ & 2 & $\left\{v_{1}, v_{4}, v_{5}\right\}$ & Yes \\
\hline$v_{4} v_{5}$ & $\begin{array}{c}\left\{v_{1}, v_{2}\right\} \\
\left\{v_{1}, v_{6}\right\},\left\{v_{1}, v_{3}\right\}\end{array}$ & 2 & $\left\{v_{1}, v_{4}, v_{5}\right\}$ & No \\
\hline$v_{5} v_{6}$ & $\left\{v_{1}, v_{2}, v_{3}, v_{4}\right\}$ & 4 & $\left\{v_{1}, v_{4}, v_{5}\right\}$ & No \\
\hline$v_{6} v_{1}$ & $\left\{v_{2}, v_{3}, v_{4}, v_{5}\right\}$ & 4 & $\left\{v_{1}, v_{4}, v_{5}\right\}$ & No \\
\hline$v_{2} v_{6}$ & $\left\{v_{1}, v_{3}, v_{4}, v_{5}\right\}$ & 4 & $\left\{v_{1}, v_{4}, v_{5}\right\}$ & No \\
\hline$v_{3} v_{5}$ & $\left\{v_{1}, v_{4}\right\}$ & 2 & $\left\{v_{1}, v_{4}, v_{5}\right\}$ & Yes \\
\hline$v_{3} v_{6}$ & $\left\{v_{1}, v_{2}, v_{4}, v_{5}\right\}$ & 4 & $\left\{v_{1}, v_{4}, v_{5}\right\}$ & No \\
\hline$v_{4} v_{6}$ & $\left\{v_{1}, v_{2}, v_{3}, v_{5}\right\}$ & 4 & $\left\{v_{1}, v_{4}, v_{5}\right\}$ & No \\
\hline
\end{tabular}


Definition 2.6. A graph $G$ is called a perfect extreme edge fixed Steiner graph if every edge $e=x y$ in $G$ has an extreme edge fixed Steiner set of $G$.

Example 2.7. Let $e=x y$ be any edge of a complete graph $K_{p}$ $(p \geq 3)$. Since every vertex of a complete graph is an extreme vertex, the extreme edge fixed Steiner set has $\mathrm{p}-2$ vertices. Therefore $K_{\mathrm{p}}(\mathrm{p} \geq 3)$ is a perfect extreme edge fixed Steiner graph for every edge $e=x y$ in $G$.

Example 2.8. For any nontrivial tree $T$ with $k$ end vertices, $\operatorname{Ext}(T)=k$ and hence any tree is an extreme edge fixed Steiner Graph with $s_{e}(T)=k$ if neither $\mathrm{x}$ nor $\mathrm{y}$ is an end vertex of T or $k-1$ if either $x$ or $y$ is an end vertex $T$. Thus any non trivial tree is a perfect extreme edge fixed Steiner graph.

Remark 2.9. In particular, a caterpillar is a perfect extreme edge fixed Steiner graph.

Remark 2.10. Any perfect extreme edge fixed Steiner graph is an extreme edge fixed Steiner graph. But not conversely, (i.e) any extreme edge fixed Steiner graph is not a perfect extreme edge fixed Steiner graph.

It is observed that the graph shown in Figure 1 is an extreme edge fixed Steiner graph but not a perfect extreme edge fixed Steiner graph.

Remark 2.11. A cycle $G$ is not an extreme edge fixed Steiner Graph for any edge e in $G$ since it has no extreme vertices.

Remark 2.12. A complete bipartite graph is not an extreme edge fixed Steiner graph for any edge e in $K_{m, n}$ with $m \leq n$ and $m+n \geq 3$ since it has no extreme vertices.

Remark 2.13. A wheel $W_{p}=K_{1}+C_{p-l}(p \geq 5)$ is not an extreme edge fixed Steiner graph for any edge e in $W_{p}$ since it has no extreme vertices. But $W_{4}$ is a perfect extreme edge fixed Steiner graph.

Theorem 2.14. The path $P_{p}$ is a perfect extreme edge fixed Steiner graph.

Proof: Let $V\left(P_{p}\right)=\left\{v_{1}, v_{2}, \ldots, v_{p}\right\}$.

Case 1: Let $e$ be an internal edge of $P_{p}$ with $p \geq 3$. By Theorem 1.1, it is obtained that the $s_{e}$ - set of $P_{p}$ contains only the end vertices $v_{1}$ and $v_{p}$. Therefore the $s_{e}$ - set of $P_{p}$ itself is the set of extreme vertices in $G$.

Case 2: Let $e$ be an end edge of $P_{p}$. Then $e=v_{1} v_{2}$ or $v_{p-1} v_{p}$. If $e=v_{1} v_{2}$, then $W=\left\{v_{p}\right\}$ is the edge fixed Steiner set of $P_{p}$ and the set of extreme vertices $S_{\text {ext }}=\left\{v_{1}, v_{p}\right\}$. Thus $W=S_{\text {ext }}-$ $\left\{v_{1}\right\}$. So $W$ is an extreme edge fixed steiner set of $P_{p}$. Similarly, if $e=v_{p-1} v_{p}$, then $\left\{v_{1}\right\}$ is the edge fixed Steiner set of $P_{p}$ which is also an extreme edge fixed steiner set of $P_{p}$. Therefore $P_{p}$ is a perfect extreme edge fixed Steiner graph.

Theorem 2.15. Let $G$ be a connected graph of order $p \geq 3$ and $e$ be any edge of $G$. Then $1 \leq \operatorname{Ext}(G)-2 \leq s_{e}(G) \leq p-2$.

Proof: It follows from Theorem 1.3.

Theorem 2.16. If a graph $G$ contains $p-2$ cut vertices or every vertex of $G$ is an extreme vertex, then $G$ is a perfect extreme edge fixed Steiner Graph.

Proof: It follows from Theorem 1.7 and from Theorem 1.3.

\section{EXTREME EDGE FIXED STEINER GRAPH AND DIAMETER OF A GRAPH}

For every connected graph $\mathrm{G}, \operatorname{rad} \mathrm{G} \leq \operatorname{diam} \mathrm{G} \leq 2 \mathrm{rad} \mathrm{G}$. Ostrand [7] proved that every two positive integers $a$ and $b$ with $\mathrm{a} \leq \mathrm{b} \leq 2 \mathrm{a}$ are realizable as the radius and diameter, respectively, of some connected graph. It is extended for the extreme edge fixed Steiner graph also as follows.

Theorem 3.1. For positive integers $r, d$ and $l \geq 2$ with $r \leq d$ $\leq 2 \mathrm{r}$, there exists a perfect extreme edge fixed Steiner graph $\mathrm{G}$ with $\operatorname{rad} \mathrm{G}=\mathrm{r}$, $\operatorname{diam} \mathrm{G}=\mathrm{d}$ and $\operatorname{se}(\mathrm{G})=\operatorname{Ext}(\mathrm{G})=1$ or $1-1$.

Proof: Let $\mathrm{e}=\mathrm{xy}$ be any edge in $\mathrm{G}$. When $\mathrm{r}=1$, we have $\mathrm{d}=1$ or 2.

If $\mathrm{d}=1$, let $\mathrm{G}=\mathrm{K} 1+2$. Then by Theorem $1.2, \operatorname{se}(\mathrm{G})=1=$ $\operatorname{Ext}(\mathrm{G})$ for any edge $\mathrm{e}=\mathrm{xy}$ in $\mathrm{G}$.

If $\mathrm{d}=2$, let $\mathrm{G}=\mathrm{K} 1,1+1$. By the Theorem $1.8, \operatorname{se}(\mathrm{G})=1$ for any edge $e=x y$ in $G$. Let $r \geq 2$. We construct a graph $G$ with required properties as follows.

Let $\mathrm{C} 2 \mathrm{r}:\{\mathrm{v} 1, \mathrm{v} 2, \ldots, \mathrm{v} 2 \mathrm{r}, \mathrm{v} 1\}$ be a cycle of order $2 \mathrm{r}$ and let $\mathrm{Pd}-\mathrm{r}+1: \mathrm{u} 0, \mathrm{u} 1, \ldots, \mathrm{ud}-\mathrm{r}$ be the path of order $\mathrm{d}-\mathrm{r}+1$. Let $\mathrm{H}$ be the graph obtained from $\mathrm{C} 2 \mathrm{r}$ and $\mathrm{Pd}-\mathrm{r}+1$ by identifying $\mathrm{v} 1$ in $\mathrm{C} 2 \mathrm{r}$ and $\mathrm{u} 0$ in $\mathrm{Pd}-\mathrm{r}+1$. Then add $(1-2)$ new vertices $\mathrm{w} 1$, $\mathrm{w} 2, \ldots, \mathrm{wl}-2$ to $\mathrm{H}$ and joining each vertex wi $(1 \leq \mathrm{i} \leq 1-2)$ to the vertex ud $-\mathrm{r}-1$ and join the vertices $\mathrm{vr}$ and $\mathrm{vr}+2$ and obtain the graph $\mathrm{G}$ which is shown in Figure 3. Now $\operatorname{rad} \mathrm{G}=$ $r$ and diam $\mathrm{G}=\mathrm{d}$ and $\mathrm{G}$ has 1 end vertices. Let $\mathrm{e}=\mathrm{u} 0 \mathrm{u} 1$ be an edge of $\mathrm{G}$. Let $\mathrm{W}=\{\mathrm{w} 1, \mathrm{w} 2, \ldots, \mathrm{wl}-2, \mathrm{ud}-\mathrm{r}, \mathrm{vr}+1\}$ be set of 1 extreme vertices of G. From the Figure 3, it is seen that $\mathrm{W}$ is an se-set of $\mathrm{G}$. It follows from the Theorem 1.3 that $\operatorname{se}(\mathrm{G})=\operatorname{Ext}(\mathrm{G})=1$. Now, let $\mathrm{e}=\mathrm{ud}-\mathrm{r}-1 \mathrm{ud}-\mathrm{r}$ be an end vertex of $\mathrm{G}$. Then $\mathrm{W}^{\prime}=\{\mathrm{w} 1, \mathrm{w} 2, \ldots, \mathrm{wl}-2, \mathrm{ud}-\mathrm{r}-1, \mathrm{vr}+1\}-$ \{ud-r.\} From the Figure 3, it is seen that W' is an se-set of G. It follows from Theorem 1.3 that $\operatorname{se}(\mathrm{G})=\operatorname{Ext}(\mathrm{G}) 1=1-1$. Therefore $\operatorname{se}(G)=\operatorname{Ext}(G)=1$ or $1-1$ for every edge $e=x y$ in $\mathrm{G}$. Thus the graph $\mathrm{G}$ in Figure 3 is a perfect extreme edge fixed Steiner graph.

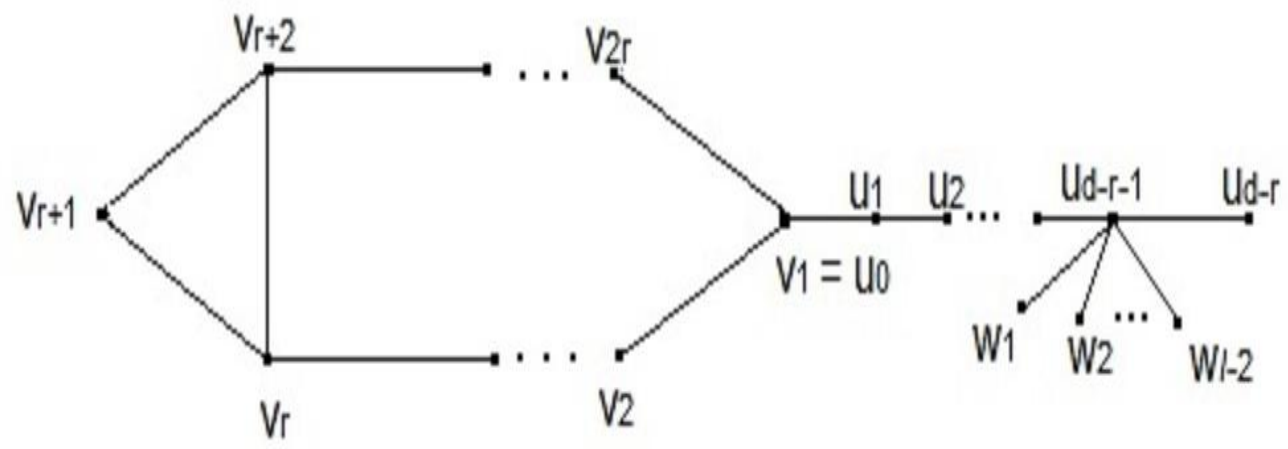

Figure 3: Constructed perfect extreme edge fixed Steiner Graph 
Theorem 3.2. For any two positive integers $a$ and $b$ with 2 $\leq a \leq b$, there exists a connected graph $G$ with $\operatorname{Ext}(G)=a$ and $s_{e}(G)=b$ for some edge $e=x y$ in $G$.

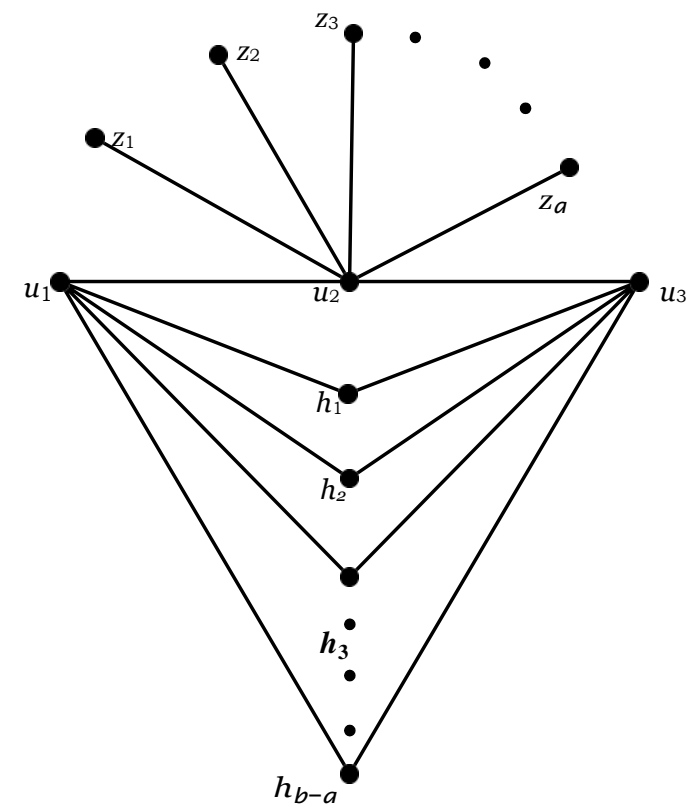

Figure 4: A graph $G$ with $\operatorname{Ext}(G)=a$ and $s_{e}(G)=b$ for some edge $e=x y$ in $G$

Proof: Let $\mathrm{G}$ be the graph obtained in Figure 4 from the path $\mathrm{P}$ on the vertices: $\mathrm{u} 1, \mathrm{u} 2, \mathrm{u} 3$, by adding the new vertices $\mathrm{z} 1$, $\mathrm{z} 2, \mathrm{za}$ and $\mathrm{h} 1, \mathrm{~h} 2, \ldots, \mathrm{hb}-\mathrm{a}$ and joining each $\mathrm{zi}(1 \leq \mathrm{i} \leq \mathrm{a})$ with $\mathrm{u} 1$ and $\mathrm{u} 3$ and also joining each hi $(1 \leq \mathrm{i} \leq \mathrm{b}-\mathrm{a})$ with $\mathrm{u} 1$ and u2. Let $\{Z=z 1, z 2, \ldots, z a\}$. Then $Z$ is the set of extreme vertices of $G$. Thus $\operatorname{Ext}(G)=a$.

Next, we prove that $\operatorname{se}(\mathrm{G})=\mathrm{b}$ for some edge e in $\mathrm{G}$. Let $\mathrm{W}$ beany edge fixed Steiner set of $\mathrm{G}$. Then by Theorem 1.3, $\mathrm{Z} \subseteq$ $W$. It is clear that $Z$ is not an edge fixed Steiner set of $G$ for any edge $\mathrm{e}=\mathrm{xy}$ in $\mathrm{G}$. Let $\mathrm{e}=\mathrm{uv}$ be an edge of G. We show that each hi $\in \mathrm{W}(1 \leq \mathrm{i} \leq \mathrm{b}-\mathrm{a})$ for the edge $\mathrm{e}=\mathrm{uv}$. Suppose that hi $\in / \mathrm{W}$ for some $\mathrm{i}(1 \leq \mathrm{i} \leq \mathrm{b}-\mathrm{a})$. Then it is clear that hi does not lie on any edge fixed Steiner $\mathrm{W}$-tree of the edge $\mathrm{e}=$ uv of $\mathrm{G}$ joining $\mathrm{e}=\mathrm{uv}$ and a vertex of $\mathrm{W}$, which is a contradiction. Therefore, each hi $(1 \leq \mathrm{i} \leq \mathrm{b}-\mathrm{a}) \in \mathrm{W}$. So $\operatorname{se}(G) \geq a+b-a=b$. hence $W=Z \cup\{h 1, h 2, \ldots, h b-a\}$ is a se-set of $G$ so that $\operatorname{se}(G)=b$.

\section{REFERENCES}

[1] B. Bollobas, Extremal Graph Theory, Academic press, 1978.

[2] F. Buckley and F. Harary,Distance in Graphs, AddisonWesley, Redwood City, CA, 1990.

[3] G. Chartrand and P. Zhang,The Steiner number of a graph, Discrete Math- ematics 242 (2002) 41 - 54 DOI: $10.1016 / \mathrm{S} 0012-365 \mathrm{X}(00) 00456-8$

[4] Gary Chatrand and Ping Zhang,Introduction to Graph Theory , Eighth Reprint 2012, Tata McGraw Hill Education Private Limited, New Delhi.F. Harary,Graph Theory, Addison-Wesley, 1969.

[5] M.Perumalsamy, P.Arul Paul Sudhahar, J.John and R.Vasanthi, Edge fixed Steiner number of a graph, International Journal of Mathematical Analysis Vol. 11, (2017), $\quad$ No. $\quad 16, \quad 771 \quad$ - 785 doi.org/10.12988/ijma.2017.7694.

[6] A.P. Santhakumaran, EXTREME STEINER GRAPHS, Discrete Mathematics, Algorithms and Applications Vol. 4, No. 2 (2012) 1250029 (11 pages)

[7] M.Perumalsamy, P.Arul Paul Sudhahar and R.Vasanthi, The Upper Edge Fixed Steiner Number of a Graph, International Journal of Mathematics And its Applications.,Volume 6(2A)(2018), 337 - 343.

[8] M.Perumalsamy, P.Arul Paul Sudhahar, R.Vasanthi and J.John, The forcing edge fixed steiner number of a graph,Journal of Statistics and Man- agement Systems, Volume 22, 2019 - Issue 1. 